\title{
PARETO OPTIMAL FEEDFORWARD CONSTRAINED REGULATION OF MIMO LINEAR SYSTEMS
}

\author{
Aurelio Piazzi* Antonio Visioli** \\ * Dipartimento di Ingegneria dell'Informazione, University \\ of Parma (Italy), e-mail: aurelio@ce.unipr.it \\ ** Dipartimento di Elettronica per l'Automazione, \\ University of Brescia, (Italy), e-mail: visioli@ing.unibs.it
}

\begin{abstract}
In this paper we present a methodology for the feedforward minimumtime regulation of Multiple-Input-Multiple-Output (MIMO) square linear systems. The aim is to synthesize bounded smooth input functions, subject to nonsaturating constraints on the inputs and their derivatives until an arbitrary predefined order, in order to provide given transitions of the outputs. The posed problem yields to the construction of a Pareto set of output transition times, which is determined by employing an ad hoc parametrized family of output transition functions and a suitably devised input-output inversion procedure. Illustrative examples are given in order to show the effectiveness of the methodology. Copyright (c) 2005 IFAC
\end{abstract}

Keywords: Feedforward control, MIMO, inversion, multiobjective optimization, constraints.

\section{INTRODUCTION}

It is well-known that the main aim of the use of feedback in a control system is to address the rejection of external disturbances and to reduce the effects of model uncertainties, whilst performances in the set-point regulation task can be improved by employing a feedforward controller. For the synthesis of feedforward actions, the fact that it is known in advance that a specified transition of the system output is required can be conveniently exploited, leading to a noncausal feedforward controller. In this context, in general, a command input with pre- and post-actuation is calculated by adopting a (stable) dynamic inversion procedure (see for example (Perez and Devasia, 2003)). From another point of view, it is also well-known that the design of a control system often involves the application of a multiobjective design technique where the improvement over some objectives might yield to the decrement in some others (Dorato, 1991). A typical example in this frame- work is the mixed $\mathcal{H}_{2} / \mathcal{H}_{\infty}$ control design problem (Khargonekar and Rotea, 1991). The solution of a multiobjective design problem is represented by a Pareto set that helps the designer to achieve the best compromise between the different objectives (Takahashi et al., 2004). In this paper we approach the problem of designing a (noncausal) feedforward controller for a MIMO linear system that is capable to provide predefined transitions of system outputs. The transition times are minimized subject to prespecified non-saturating constraints on the system inputs and their derivatives until a predefined order. The Pareto set that represents the solution of this multiobjective optimization problem (MOP) is calculated by employing a stable input-output dynamic inversion procedure, after that the desired output functions have been selected as a family of parameterised polynomials. Notation. $\mathbb{R}_{+}$denotes the set of positive real numbers. $C^{i}$ denotes the set of scalar real functions that are continuous till the $i$ th derivative. The $i$ th order differential operator is $D^{i}$. If $p(t)$ is a poly- 
nomial with argument $t$ then $\operatorname{deg}\{p(t)\}$ denotes its degree. Bold capital letters are adopted to denote matrices or vectors obtained by extracting a row or a column of a matrix, whilst bold lower-case letters are employed to denote vectors. Elements of a matrix or of a vector are denoted indifferently with capital or lower-case italic letters.

\section{PROBLEM FORMULATION}

We consider a linear, time-invariant, continuoustime asymptotically stable square MIMO system $\Sigma$ whose matrix transfer function is:

$$
\mathbf{H}(s)=\left[H_{i j}(s)\right] \quad i=1, \ldots, p \quad j=1, \ldots, p
$$

where

$$
\begin{aligned}
& H_{i j}(s)=K_{i j} \frac{b_{i j}(s)}{a_{i j}(s)} \\
& =K_{i j} \frac{s^{m_{i j}}+b_{i j, m_{i j}-1} s^{m_{i j}-1}+\cdots+b_{i j, 0}}{s^{n_{i j}}+a_{i j, n_{i j}-1} s^{n_{i j}-1}+\cdots+a_{i j, 0}}
\end{aligned}
$$

are strictly proper, rational transfer functions where no pole-zero cancellations occur (i.e. $b_{i j}(s)$ and $a_{i j}(s)$ are coprime). In order to assure the invertibility of the system and to pose a sensible regulation problem we assume that $H(s)$ has a full normal rank (i.e. the normal rank of $H(s)$ is equal to $p$ ) and that $\Sigma$ has not purely imaginary zeros. The input and output vectors of $\Sigma$ are $\mathbf{u} \in \mathbb{R}^{p}$ and $\mathbf{y} \in \mathbb{R}^{p}$ respectively and the set of all the cause/effect vectors associated with $\Sigma$ (i.e. the behavior set of $\Sigma$ (Polderman and Willems, 1998)) is denoted by

$$
\begin{aligned}
& \mathcal{B}:=\left\{(\mathbf{u}(\cdot), \mathbf{y}(\cdot)) \in P_{c} \times P_{c}:\right. \\
& (\mathbf{u}(\cdot), \mathbf{y}(\cdot)) \text { is a weak solution of } \\
& {\left[\begin{array}{l}
\sum_{i=0}^{n_{11}} a_{11, i} D^{i} y_{1}+\cdots+\sum_{i=0}^{n_{1 p}} a_{1 p, i} D^{i} y_{p} \\
\vdots \\
\sum_{i=0}^{n_{p 1}} a_{p 1, i} D^{i} y_{1}+\cdots+\sum_{i=0}^{n_{p p}} a_{p p, i} D^{i} y_{p}
\end{array}\right]=} \\
& {\left[\begin{array}{l}
\sum_{i=0}^{m_{11}} b_{11, i} D^{i} u_{1}+\cdots+\sum_{i=0}^{m_{1 p}} b_{1 p, i} D^{i} u_{p} \\
\vdots \\
\sum_{i=0}^{m_{p 1}} b_{p 1, i} D^{i} u_{1}+\cdots+\sum_{i=0}^{m_{p p}} b_{p p, i} D^{i} u_{p}
\end{array}\right]}
\end{aligned}
$$

where $P_{c}$ denotes the set of piecewise continuous functions defined over $(-\infty,+\infty)$, i.e. the real field $\mathbb{R}$. The concept of weak solution has been introduced by Polderman and Willems in their behavioral approach to system theory (Polderman and Willems, 1998).

The considered regulation problem consists of obtaining, for each system output $y_{i}, i=1, \ldots, p$, a transition from a previous set-point value $y_{i}^{0}$ to a new one $y_{i}^{1}$. In the following, without loss of generality, it will be assumed $y_{i}^{0}=0, i=$ $1, \ldots, p$. The authors have shown in (Piazzi and A.Visioli, 2001; Piazzi and Visioli, 2001a; Piazzi and Visioli, 2001b) (for SISO systems) that, for set-point regulation problems, a good choice for the $i$ th output time function is the following transition function $y_{i}\left(t ; \tau_{i}\right), i=1, \ldots, p$ defined as

$$
\begin{aligned}
& y_{i}\left(t ; \tau_{i}\right)=
\end{aligned}
$$

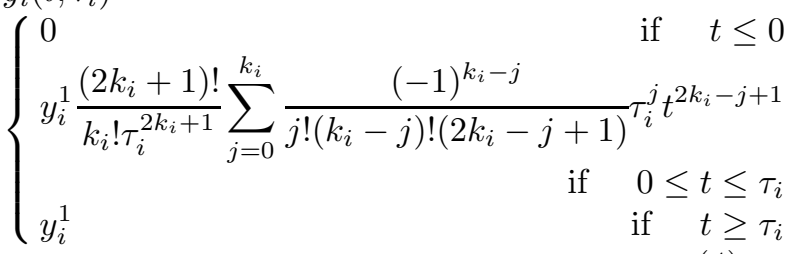

Function $y_{i}\left(t ; \tau_{i}\right)$, parameterised by the transition time $\tau_{i}$ is a $C^{k_{i}}$-function over $(-\infty,+\infty)$ and is strictly increasing in the interval $\left[0, \tau_{i}\right]$ so that neither overshooting nor undershooting appear in this output planning for set-point regulation. The choice of the design parameter $k_{i}$ will be discussed in subsection 3.1.

In this context, the following Pareto optimal feedforward constrained regulation problem emerges:

(1) Determine a vector of input functions $\mathbf{u}(t ; \boldsymbol{\tau})$ bounded over $(-\infty,+\infty)$ such that

$$
(\mathbf{u}(\cdot, \boldsymbol{\tau}), \mathbf{y}(\cdot, \boldsymbol{\tau})) \in \mathcal{B}
$$

where $\mathbf{y}(\cdot, \boldsymbol{\tau}):=\left[y_{1}\left(\cdot ; \tau_{1}\right), \ldots, y_{p}\left(\cdot ; \tau_{p}\right)\right]^{T}$.

(2) Determine the transition times vector $\boldsymbol{\tau}=$ $\left[\tau_{1}, \ldots, \tau_{p}\right]^{T}$ by solving the following MOP:

$$
\min _{\boldsymbol{\tau} \in \mathbb{R}_{+}^{p}} \boldsymbol{\tau}
$$

such that, $i=1, \ldots, p, j=0,1, \ldots, l_{i}$,

$$
\left|D^{j} u_{i}(t ; \boldsymbol{\tau})\right| \leq u_{M, i}^{(j)} \quad \forall t \in(-\infty,+\infty)
$$

The positive values $u_{M, i}^{(j)}, j=0,1, \ldots, l_{i}$, $i=1, \ldots, p$, are given bounds of the problem.

We propose to solve step 1 of the above problem by adopting a stable (multi-)input-(multi-)output dynamic inversion procedure, that allows to determine a closed-form expression of the command inputs vector which renders the solution of step 2 quite straightforward.

\section{STABLE INPUT-OUTPUT INVERSION}

\subsection{Choice of the output functions order}

The choice of the order $k_{i}$ of the $i$ th polynomial output function can be done by considering a somewhat trivial extension of a property reported in (Piazzi and Visioli, 2001a). The inverse of $\mathbf{H}(s)$ can be expressed as follows:

$$
\mathbf{H}^{-1}(s)=\mathbf{F}(s)+\mathbf{H}_{0}(s)
$$

where $\mathbf{F}(s)=\left[F_{i j}(s)\right], i=1, \ldots, p, j=1, \ldots, p$ is a matrix whose elements are polynomials and $\mathbf{H}_{0}(s)$ is a matrix of strictly proper rational transfer functions. Denote by $\rho_{i j}$ the order of the polynomial $F_{i j}(s)$. Then, we have the following property.

Property 1. Consider $(\mathbf{u}(\cdot), \mathbf{y}(\cdot)) \in \mathcal{B}$. Then, $u_{i}(\cdot) \in C^{l_{i}}$ if $y_{j}(\cdot) \in C^{l_{i}+\rho_{i j}}, j=1, \ldots, p$. 
Actually, the choice of the order $k_{j}$ of the output function $y_{j}\left(t ; \tau_{j}\right)$ has to be done in such a way that the input functions are continuous up to the $l_{i}$ th order so that condition (7) is well-posed. Hence, we have to select

$$
k_{j}=\max _{i=1, \ldots, p}\left\{\rho_{i j}+l_{i}\right\}, \quad j=1, \ldots, p
$$

Remark 1. The statement of Property 1 also holds with $l_{i}=-1$ provided that conventionally $C^{-1}(\mathbb{R})$ coincides with $P_{c}$, i.e. the set of piecewise continuous functions defined over $\mathbb{R}$.

\subsection{Command inputs synthesis}

Once the parameterised output functions vector has been defined, the following (multi-)input(multi-)output stable dynamic inversion procedure has to be performed (extending that devised for the SISO case (Piazzi and Visioli, 2005)). Starting from (8), the inverse of the transfer function matrix $\mathbf{H}(s)$ can be expressed as $(\rho:=$ $\max \left\{\rho_{1}, \ldots, \rho_{p}\right\}$ where $\left.\rho_{i}:=\max \left\{\rho_{1 i}, \ldots, \rho_{p i}\right\}\right)$

$$
\mathbf{H}^{-1}(s)=\boldsymbol{\Gamma}_{\rho} s^{\rho}+\cdots+\boldsymbol{\Gamma}_{0}+\mathbf{H}_{0}(s)
$$

where $\boldsymbol{\Gamma}_{i}, i=0, \ldots, \rho$ is a matrix of scalar elements. Then, we can also express $\mathbf{H}_{0}(s)$ as

$$
\mathbf{H}_{0}(s)=\frac{\mathbf{C}(s)}{z_{H}(s)}
$$

where $z_{H}(s)$ is the zero polynomial of $\Sigma$. Polynomial $z_{H}(s)$ can be factorized as

$$
z_{H}(s)=z_{H}^{-}(s) z_{H}^{+}(s)
$$

where $z_{H}^{-}(s)$ and $z_{H}^{+}(s)$ denote the polynomials associated to the zeros of $\Sigma$ with negative and positive real part respectively. Thus, by using the partial fraction expansion, $\mathbf{H}_{0}(s)$ can be decomposed into stable and unstable parts according to

$$
\mathbf{H}_{0}(s)=\mathbf{H}_{0}^{-}(s)+\mathbf{H}_{0}^{+}(s)=\frac{\mathbf{D}(s)}{z_{H}^{-}(s)}+\frac{\mathbf{E}(s)}{z_{H}^{+}(s)}
$$

where, having defined $m^{-}:=\operatorname{deg}\left\{z_{H}^{-}(s)\right\}, m^{+}:=$ $\operatorname{deg}\left\{z_{H}^{+}(s)\right\}$, we have $\operatorname{deg}\left\{D_{i j}(s)\right\} \leq m^{-}-1$ and $\operatorname{deg}\left\{E_{i j}(s)\right\} \leq m^{+}-1, i=1, \ldots, p, j=1, \ldots, p$. The Laplace transform of the transition functions vector be denoted by $\tilde{\mathbf{y}}(s ; \boldsymbol{\tau}):=\mathcal{L}[\mathbf{y}(t ; \boldsymbol{\tau})]$; then a vector of command input signals that causes the desired $\mathbf{y}(t ; \boldsymbol{\tau})$ on the output of $\Sigma$ can be determined by the standard input-output dynamic inversion:

$$
\mathbf{u}_{u}(t ; \boldsymbol{\tau}):=\mathcal{L}^{-1}\left[\mathbf{H}^{-1}(s) \tilde{\mathbf{y}}(s ; \boldsymbol{\tau})\right] .
$$

If the elements of $\mathbf{y}(t ; \boldsymbol{\tau})$ are sufficiently smooth (see Property 1$)$ then evidently $\left(\mathbf{u}_{u}(\cdot ; \boldsymbol{\tau}), \mathbf{y}(\cdot, \boldsymbol{\tau})\right) \in$ $\mathcal{B}$ but unfortunately in general $\mathbf{u}_{u}(t ; \boldsymbol{\tau})$ is unbounded over $[0,+\infty)$ due to the presence of the unstable zero dynamics. Nevertheless, the knowledge of the structure of $\mathbf{u}_{u}(t ; \boldsymbol{\tau})$ can be exploited to solve the stable dynamic inversion problem.

Define $\eta_{i j}^{-}(t):=\mathcal{L}^{-1}\left[H_{0, i j}^{-}(s)\right]$ and $\eta_{i j}^{+}(t):=$
$\mathcal{L}^{-1}\left[H_{0, i j}^{+}(s)\right]$ and from (10)-(14) it follows that $\mathbf{u}_{u}(t ; \boldsymbol{\tau})=\mathbf{0}$ if $t<0$ and if $t \geq 0(i=1, \ldots, p)$ :

$$
\begin{aligned}
u_{u, i}(t ; \boldsymbol{\tau})= & \sum_{j=1}^{p} \Gamma_{\rho, i j} D^{\rho} y_{j}\left(t ; \tau_{j}\right)+\cdots \\
& \cdots+\sum_{j=1}^{p} \Gamma_{0, i j} D^{\rho} y_{j}\left(t ; \tau_{j}\right) \\
& +\sum_{j=1}^{p} \int_{0}^{t} \eta_{i j}^{-}(t-v) y_{j}\left(v ; \tau_{j}\right) d v \\
& +\sum_{j=1}^{p} \int_{0}^{t} \eta_{i j}^{+}(t-v) y_{j}\left(v ; \tau_{j}\right) d v
\end{aligned}
$$

The modes associated to $z_{H}^{+}(s)$ be denoted by $m_{i}^{+}(t), i=1 \ldots, m^{+}$. Then, expression (15) can be rewritten as (see (Piazzi and Visioli, 2005))

$$
\begin{aligned}
& u_{u, i}(t ; \boldsymbol{\tau})=\sum_{j=1}^{p} \Gamma_{\rho, i j} D^{\rho} y_{j}\left(t ; \tau_{j}\right)+\cdots+ \\
& \sum_{j=1}^{p} \Gamma_{0, i j} D^{\rho} y_{j}\left(t ; \tau_{j}\right)+\sum_{j=1}^{p} \int_{0}^{t} \eta_{i j}^{-}(t-v) y_{j}\left(v ; \tau_{j}\right) d v \\
& +f_{i}(t)+\sum_{j=1}^{m^{+}} \mu_{i j} m_{j}^{+}(t)
\end{aligned}
$$

where function $f_{i}(t)$ is bounded over $[0,+\infty)$ and $\mu_{i 1}, \ldots, \mu_{i m^{+}}$are appropriate coefficients. Taking into account expression (16) we define the "correcting" $i$ th input over the whole time axis as

$$
u_{c, i}(t ; \boldsymbol{\tau})=-\sum_{j=1}^{m^{+}} \mu_{i j} m_{j}^{+}(t)
$$

Property 2. Having defined as in (17) the inputs vector $\mathbf{u}_{c}(t ; \boldsymbol{\tau})=\left[u_{c, 1}(t ; \boldsymbol{\tau}), \ldots, u_{c, 1}(t ; \boldsymbol{\tau})\right]^{T}$ we have

$$
\left(\mathbf{u}_{c}(\cdot ; \boldsymbol{\tau}), \mathbf{0}\right) \in \mathcal{B} \quad \forall \boldsymbol{\tau} \in \mathbb{R}_{+}^{p}
$$

Proof. Omitted for brevity. It is based on the coefficient annihilating property for which it is $\left[\mu_{1, j}, \ldots, \mu_{p, j}\right]^{T} \in \operatorname{Ker} H\left(z_{j}\right)$ where $z_{j}$ is the unstable zero associated to $m_{j}^{+}(t)$.

Thus, the solution to the stable inversion problem (step 1 in Section 2) is provided by the following input signals vector:

$\mathbf{u}(t ; \boldsymbol{\tau})=\mathbf{u}_{u}(t ; \boldsymbol{\tau})+\mathbf{u}_{c}(t ; \boldsymbol{\tau}) \quad t \in(-\infty,+\infty)$

Taking into account (16) and (17) the following closed-form expression of the $i$ th command input signal results :

$$
\begin{aligned}
& u_{i}(t ; \boldsymbol{\tau})=-\sum_{j=1}^{m^{+}} \mu_{i j} m_{j}^{+}(t) \quad \text { if } \quad t<0 \\
& u_{i}(t ; \boldsymbol{\tau})=\sum_{j=1}^{p} \Gamma_{\rho, i j} D^{\rho} y_{j}\left(t ; \tau_{j}\right)+\cdots+ \\
& \sum_{j=1}^{p} \Gamma_{0, i j} D^{\rho} y_{j}\left(t ; \tau_{j}\right)+\sum_{j=1}^{p} \int_{0}^{t} \eta_{i j}^{-}(t-v) y_{j}\left(v ; \tau_{j}\right) d v \\
& +f_{i}(t) \quad \text { if } \quad t \geq 0
\end{aligned}
$$


Property 3. Assume that $y_{i}(\cdot) \in C^{\rho_{i}-1}, i=$ $1, \ldots, p$. Then, for any $\boldsymbol{\tau}>\mathbf{0}$ function $\mathbf{u}(t ; \boldsymbol{\tau})$ defined in (19) is bounded over $(-\infty,+\infty)$ and $\left(\mathbf{u}_{c}(\cdot ; \boldsymbol{\tau}), \mathbf{y}(\cdot ; \boldsymbol{\tau})\right) \in \mathcal{B}$

Proof. Omitted for brevity.

Remark 2. It is worth underlying that the determination of the command signals can be easily performed via symbolic computation by means of state-of-the-art analytical computation software, with the advantage of the avoidance of numerical difficulties (though some problems might emerge for large-scale systems).

\section{MULTIOBJECTIVE OPTIMIZATION PROBLEM}

In order to adopt the command inputs obtained by applying the input-output inversion procedure of Section 3 for the purpose of set-point regulation, suitable non-saturating constraints have to be applied on the inputs and their derivatives. Typically, constraints on the inputs and their first derivatives are of concern in practical applications, however in the devised framework we can impose constraints on the derivatives until an arbitrary order, as denoted by expression (7). Thus, to provide a minimum-time solution of the feedforward constrained regulation problem we have to solve the MOP (6)-(7). In order for this problem to be well-posed, function $D^{l_{i}} u_{i}(t ; \boldsymbol{\tau})$ has to be continuous and this is guaranteed by a suitable choice of the order of the output functions according to Property 1 and expression (9). Then, the following (mild) condition on the selected constraints has to be verified.

Proposition 1. The MOP (6)-(7) has a solution if

$$
\begin{aligned}
& \mathbf{u}_{M}^{(0)}>\mathbf{H}^{-1}(0) \mathbf{y}^{1} \quad \text { and } \quad u_{M, i}^{(j)}>0 \\
& i=1, \ldots, p \quad j=1, \ldots, l_{i}
\end{aligned}
$$

where $\mathbf{u}_{M}^{(0)}:=\left[u_{M, 1}^{(0)}, \ldots, u_{M, p}^{(0)}\right]^{T}$.

Proof. It is a simple extension of a proof appeared in (Piazzi and Visioli, 2001b).

It is worth noting that the sufficient conditions (21) are also necessary ones in practice. Indeed, if any of the conditions (21) fails to be satisfied, the feedforward constrained regulation of system (1) is almost impossible regardless of the chosen planned output transition function (for example, the control input can not sustain the constant desired output in the system steady-state conditions). Thus, the feasible set of the MOP (6)-(7) be denoted as

$$
\begin{array}{r}
\mathcal{F}:=\left\{\boldsymbol{\tau} \in \mathbb{R}_{+}^{p}:\left|D^{j} u_{i}(t ; \boldsymbol{\tau})\right| \leq u_{M, i}^{(j)} \forall t \in \mathbb{R},\right. \\
\left.i=1, \ldots, p, \quad j=0, \ldots, l_{i}\right\} .
\end{array}
$$

Denoting by $\partial \mathcal{F}$ the boundary of the feasible set $\mathcal{F}$ we have that the Pareto set that is the solution of the addressed MOP is

$$
\begin{aligned}
& \mathcal{P}:=\left\{\boldsymbol{\tau}^{*} \in \partial \mathcal{F}: \nexists \boldsymbol{\tau} \in \partial \mathcal{F}:\right. \\
& \left.\left\{\boldsymbol{\tau} \leq \boldsymbol{\tau}^{*} \text { and } \exists \tau_{i}: \tau_{i}<\tau_{i}^{*}\right\}\right\} \text {. }
\end{aligned}
$$

Actually, having the closed-form expression of the parameterised system inputs provided by the devised dynamic inversion procedure of Section 3 allows to find the Pareto set (23) quite straightforwardly by adopting a standard multiobjective optimization procedure such as the $\varepsilon$-constraint method (Miettinen, 1999). Note that the set is not convex in general (see Section 5).

\section{ILLUSTRATIVE EXAMPLES}

From a practical point of view, in order to use the synthesized minimum-time input functions (20) it is necessary to truncate them, resulting therefore in an approximate generation of the desired outputs $\mathbf{y}\left(t ; \boldsymbol{\tau}^{*}\right)$. However, this can be done with arbitrary precision given any couple of small parameters $\varepsilon_{0}>0$ and $\varepsilon_{1}>0$. Indeed, compute

$t_{0}:=\max \left\{t^{\prime} \in \mathbb{R}:\left|\mathbf{u}\left(t ; \boldsymbol{\tau}^{*}\right)\right| \leq \varepsilon_{\mathbf{0}} \quad \forall t \in\left(-\infty, t^{\prime}\right]\right\}$ where $\varepsilon_{0}:=\left[\varepsilon_{0}, \ldots, \varepsilon_{0}\right]$ and define $t_{s}:=\min \left\{0, t_{0}\right\}$. Similarly, compute

$$
t_{1}:=\min \left\{t^{\prime} \in \mathbb{R}: \underset{\left.\forall t \in\left[t^{\prime}, \infty\right)\right\}}{\left|\mathbf{u}\left(t ; \boldsymbol{\tau}^{*}\right)-\mathbf{H}^{-1}(0) \mathbf{y}^{1}\right| \leq \varepsilon_{\mathbf{1}}}\right.
$$

where $\varepsilon_{1}:=\left[\varepsilon_{1}, \ldots, \varepsilon_{1}\right]$ and define $t_{f}:=$ $\max \left\{\tau_{1}^{*}, \ldots, \tau_{p}^{*}, t_{1}\right\}$.

Thus, the approximate input signal to be actually used is

$$
\mathbf{u}_{a}\left(t ; \boldsymbol{\tau}^{*}\right):= \begin{cases}0 & \text { for } t<t_{s} \\ \mathbf{u}\left(t ; \boldsymbol{\tau}^{*}\right) & \text { for } t_{s} \leq t \leq t_{f} \\ \mathbf{H}^{-1}(0) \mathbf{y}^{1} & \text { for } t>t_{f}\end{cases}
$$

Note that typically $t_{s}<0$ and $t_{f}>\max \left\{\tau_{1}^{*}, \ldots, \tau_{p}^{*}\right\}$, associated to the pre-action and post-action control respectively.

As a first example we consider the Rosenbrock's benchmark system (Rosenbrock, 1970), which is a system that, despite its apparent simplicity, it is difficult to control due to the presence of an unstable zero. Its transfer function is:

$$
\mathbf{H}(s)=\left[\begin{array}{cc}
\frac{1}{s+1} & \frac{2}{s+3} \\
\frac{1}{s+1} & \frac{1}{s+1}
\end{array}\right]
$$

Then, we set $\mathbf{y}^{1}=\left[\begin{array}{ll}1 & 4\end{array}\right]^{T}$ and the following constraints on the input functions and their first time derivatives are considered: $u_{M, 1}^{(0)}=u_{M, 2}^{(0)}=20$ and $u_{M, 1}^{(1)}=u_{M, 2}^{(1)}=40$ (note that $l_{1}=l_{2}=1$ ). Thus, according to expression (9), we have $k_{1}=$ $k_{2}=2$ and therefore (see (4))

$y_{i}\left(t ; \tau_{i}\right)=6 \frac{t^{5}}{\tau_{i}^{5}}-15 \frac{t^{4}}{\tau_{i}^{4}}+10 \frac{t^{3}}{\tau_{i}^{3}}, \quad t \in\left[0, \tau_{i}\right] \quad i=1,2$

By evaluating (8)-(13), we obtain:

$\mathbf{H}^{-1}(s)=\left[\begin{array}{cc}-s-5 & 2 s+6 \\ s+5 & -s-5\end{array}\right]+\left[\begin{array}{cc}-\frac{8}{s-1} & \frac{8}{s-1} \\ \frac{8}{s-1} & -\frac{8}{s-1}\end{array}\right]$ 


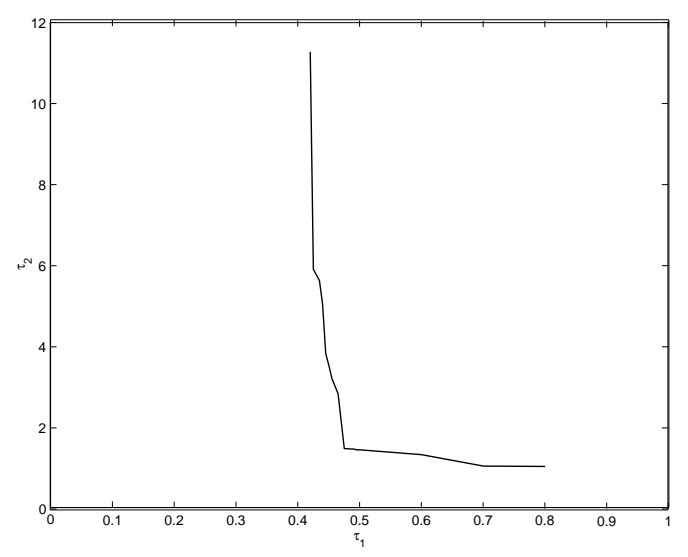

Fig. 1. Pareto set obtained for Rosenbrock system.

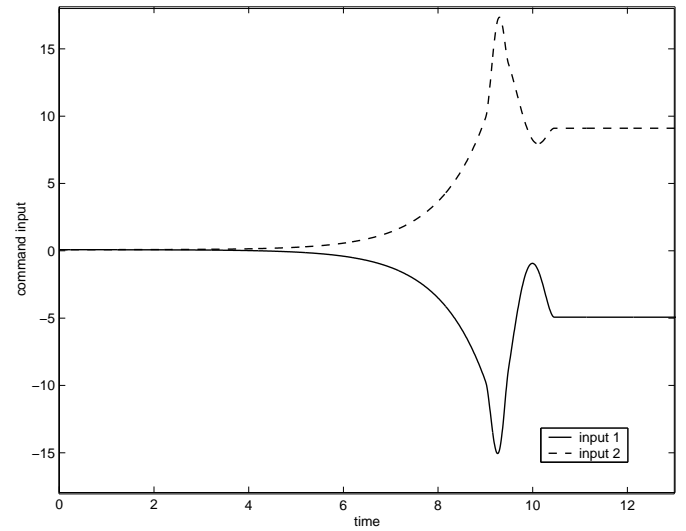

Fig. 2. Command inputs obtained for Rosenbrock system.

Thus,

$$
\mathbf{H}_{0}(s)=\mathbf{H}_{0}^{+}(s)=\frac{\left[\begin{array}{cc}
-8 & 8 \\
8 & -8
\end{array}\right]}{z_{H}^{+}(s)}
$$

where, evidently, $z_{H}^{+}(s)=s-1$ (note that $m^{+}=$ $1)$. Details on the synthesized input functions are omitted for brevity. By applying the multiobjective optimization procedure, the (non-convex) Pareto set $\mathcal{P}$ shown in Figure 1 has been obtained. As an example, we consider the case $\boldsymbol{\tau}=$ [0.48 1.45]. By setting $\varepsilon_{0}=0.001$ we obtain a preaction time $t_{s}=-9$. Note that there is no post-actuation due to the absence of a stable zero dynamics. Plots of the resulting command inputs and their first derivatives are shown in Figures 2 and 3 respectively. The corresponding system outputs are plotted in Figure 4. Note that, for the sake of clarity, the time axis has been conveniently shifted in order to have $t_{s}=0$.

As a second example we consider the following system, with one real stable zero and two complex conjugate unstable zeros:

$\mathbf{H}(s)=\left[\begin{array}{cc}2 \frac{s^{2}-2 s+5}{(s+2)(s+4)^{2}} & \frac{s^{2}-2 s+5}{(s+2)(s+3)^{2}} \\ 3 \frac{s^{2}-2 s+5}{(s+2)^{2}(s+4)} & \frac{s^{2}-2 s+5}{(s+2)^{2}(s+3)}\end{array}\right]$

In this case we set $\mathbf{y}^{1}=\left[\begin{array}{ll}2 & 1\end{array}\right]^{T}$ and the following constraints on the input functions and their

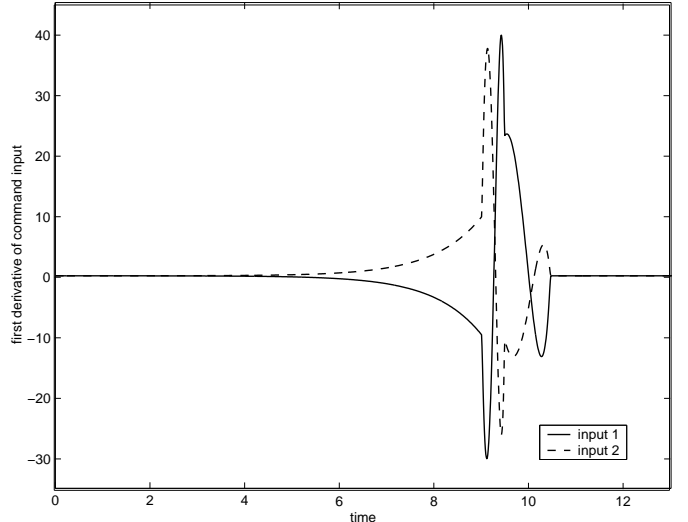

Fig. 3. First derivatives of the command inputs obtained for Rosenbrock system.

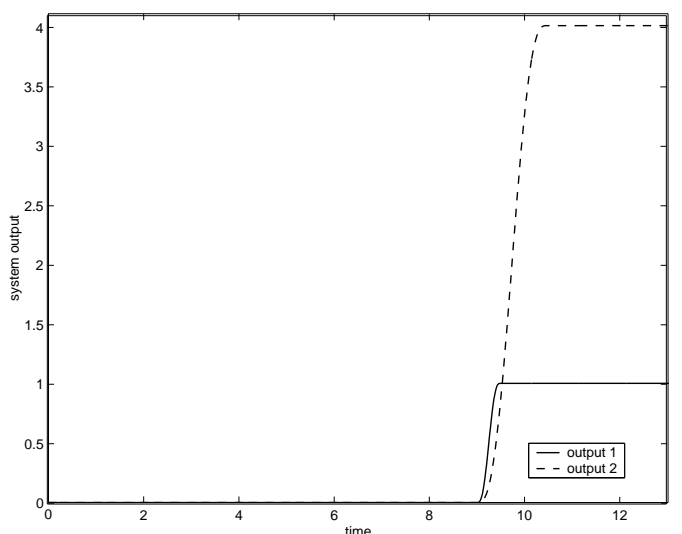

Fig. 4. Outputs obtained for Rosenbrock system.

first time derivatives are considered: $u_{M, 1}^{(0)}=10$, $u_{M, 2}^{(0)}=20$ and $u_{M, 1}^{(1)}=15, u_{M, 2}^{(1)}=30$ (note that $\left.l_{1}=l_{2}=1\right)$. Thus, as in the first example, we have $k_{1}=k_{2}=2$ and therefore we have the same output functions (25).

The inverse of $\mathbf{H}(s)$ can be expressed as (see (8) and (11))

$$
\mathbf{H}^{-1}(s)=\mathbf{F}(s)+\frac{1}{z_{H}^{+}(s) z_{H}^{-}(s)} \mathbf{C}(s)
$$

where

$$
\mathbf{F}(s)=\left[\begin{array}{cc}
-s-9 & s+8 \\
3 s+24 & -s-5
\end{array}\right]
$$

$\mathbf{C}(s)=\left[\begin{array}{cc}-33 s^{2}-31 s+174 & 27 s^{2}+122 s-176 \\ 84 s^{2}+384 s-504 & -40 s^{2}-144 s-288\end{array}\right]$

and

$$
z_{H}^{-}(s)=s+1 \quad z_{H}^{+}(s)=s^{2}-2 s+5 .
$$

Details on the synthesized input functions are omitted again for brevity. By applying the multiobjective optimization procedure, the (nonconvex and disjoint) Pareto set $\mathcal{P}$ shown in Figure 5 as a thick line has been obtained. For the sake of clarity also the boundary $\partial \mathcal{F}$ of the feasible set has been plotted (as a thin line). As an example, we consider the case $\boldsymbol{\tau}=\left[\begin{array}{ll}2 & 0.93\end{array}\right]$. By setting $\varepsilon_{0}=\varepsilon_{1}=0.001$ we obtain a preaction time $t_{s}=-9.35$ and a postaction time of $t_{f}=0.8$. Plots of the resulting command inputs and their first derivatives are shown in Figures 6 and 7 


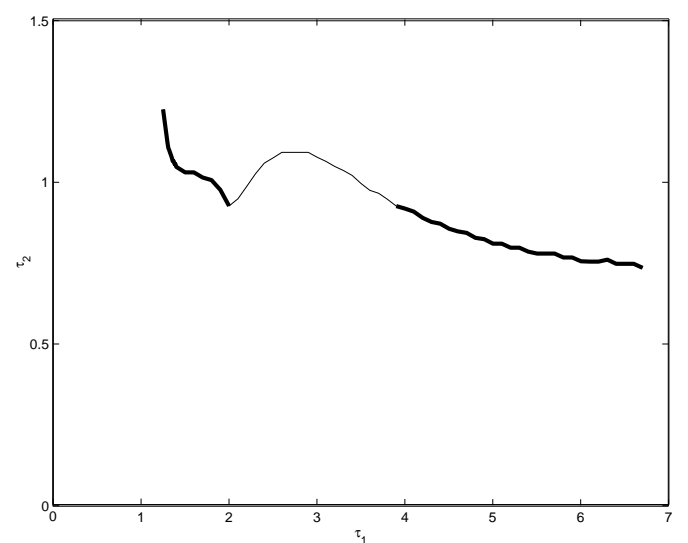

Fig. 5. Thick line: Pareto set obtained for the second example. Thin line: boundary of the feasible set.

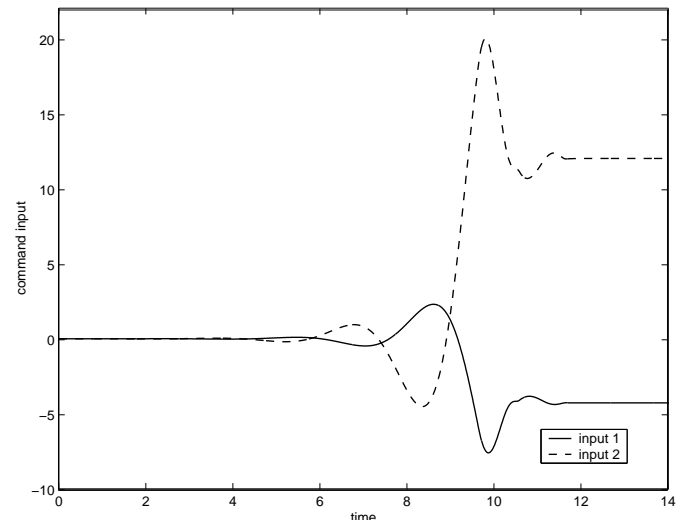

Fig. 6. Command inputs obtained for the second example.

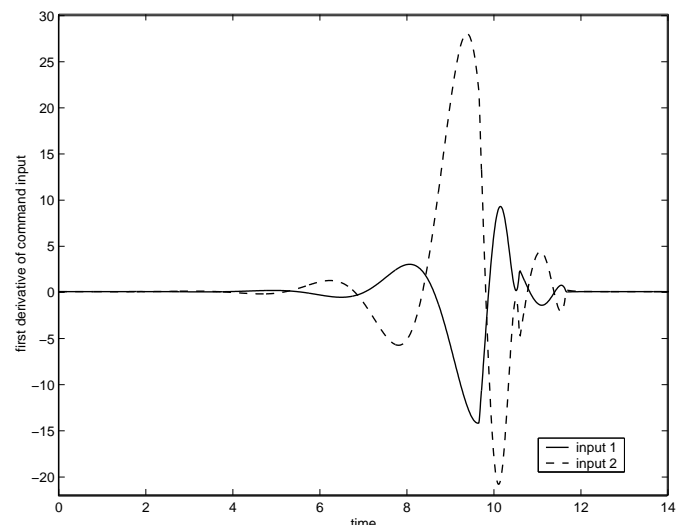

Fig. 7. First derivatives of the command inputs obtained for the second example.

respectively. The corresponding system outputs are plotted in Figure 8. As in the first example, for the sake of clarity, the time axis has been conveniently shifted in order to have $t_{s}=0$.

\section{CONCLUSIONS}

In this paper we have presented an inversionbased method for the solution of the minimumtime feedforward constrained regulation of square MIMO linear systems. A key role in the technique is played by the adoption of the "transition"

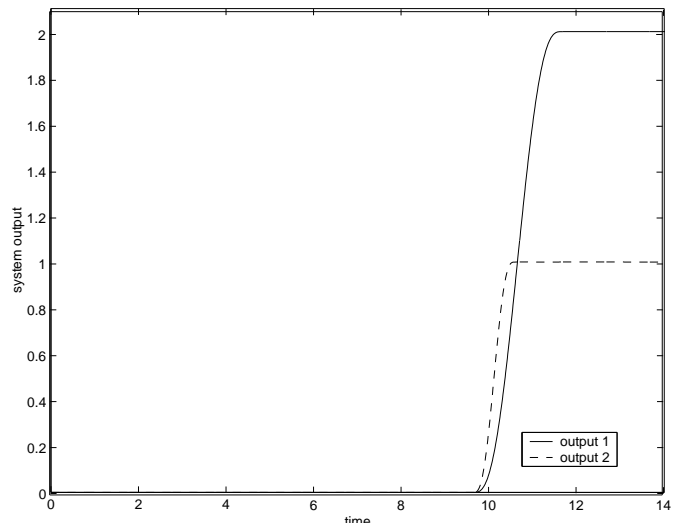

Fig. 8. Outputs obtained for the second example. polynomials as desired output functions, as they allow to obtain arbitrarily smooth input functions so that constraints on the inputs derivatives until a predefined order can be addressed. Further, they allow to determine closed-form expressions of the parameterised input signals so that the Pareto set, which is the solution of the MOP, can be calculated with standard procedures. The methodology can be readily adopted to improve the performances of feedback regulators.

\section{REFERENCES}

Dorato, P. (1991). A survey of robust multiobjective design techniques. In: Control of Uncertain Dynamic Systems (S. P. Battacharyya and L. H. Kell eds). CRC Press. pp. 249-259.

Khargonekar, P. P. and M. A. Rotea (1991). Mixed $\mathcal{H}_{2} / \mathcal{H}_{\infty}$ control: a convex optimization approach. IEEE TAC 36, 824-837.

Miettinen, K. M. (1999). Nonlinear Multiobjective Optimization. Kluwer Academic Publisher.

Perez, H. and S. Devasia (2003). Optimal output transitions for linear systems. Automatica 39, 181-192.

Piazzi, A. and A. Visioli (2001a). Optimal noncausal set-point regulation of scalar systems. Automatica 37, 121-127.

Piazzi, A. and A. Visioli (2001b). Robust set-point constrained regulation via dynamic inversion. Int. J. of Robust and Nonlin. Cont. 11, 1-22.

Piazzi, A. and A. Visioli (2005). Using stable input-output inversion for minimum-time feedforward constrained regulation of scalar systems. Automatica 41, 305-313.

Piazzi, A. and A.Visioli (2001). Optimal inversionbased control for the set-point regulation of nonminimum-phase uncertain scalar systems. IEEE Trans. on Aut. Cont. 46, 1654-1659.

Polderman, J. W. and J. C. Willems (1998). Introduction to Mathematical Systems Theory. Springer-Verlag. New York, USA.

Rosenbrock, H. H. (1970). State-Space and Multivariable Theory. Nelson. London, UK.

Takahashi, R. H. C., R. M. Palhares, D. A. Dutra and L. P. S. Goncalves (2004). Estimation of Pareto sets in the mixed $\mathcal{H}_{2} / \mathcal{H}_{\infty}$ control problem. Int. J. of Syst. Science 35, 55-67. 